\title{
Application of neural networks to lighting systems
}

\author{
Marek Dudzik ${ }^{1}$, Mirosław Dechnik ${ }^{2,3,{ }^{*}, \text { Marcin }_{\text {Furtak }}{ }^{2}}$ \\ ${ }^{1}$ Cracow University of Technology, Faculty of Electrical and Computer Engineering, Institute of \\ Electrical Engineering and Computer Science \\ ${ }^{2}$ Cracow University of Technology, Faculty of Civil Engineering, Małopolska Laboratory of Energy \\ Efficient Building (MLBE) \\ ${ }^{3}$ AGH University of Science and Technology, Faculty of Electrical Engineering, Automatics, \\ Computer Science and Biomedical Engineering
}

\begin{abstract}
This paper will continue application of neural networks to the concept of environmental quality management technology described elsewhere. Human preferences are different and may vary depending on type of the work or psycho-physical conditions of workers. This paper deals with application of artificial neural networks (ANNs) to control of general lighting systems to provide personal (individual) illuminance on worktables. Two-layer feedforward ANN is used to identify and model the system. Introduction of ANN to model illumination systems opens a new possibility of individual control systems in which specific areas have different set of requirements than remaining areas of a lighting system.
\end{abstract}

\section{Introduction}

It is estimated that lighting is responsible for $20-40 \%$ [1] of total energy consumption in buildings. Among the existing sources of light, highly-efficient LED sources have been particularly popular in recent years. Because the dimming of LED lamps causes a proportional reduction in their energy consumption [2], their use in energy-saving lighting control systems using dimming method enables significant energy savings.

Artificial intelligence (AI) has been used in lighting prediction and lighting control in office space for only two decades [3]. Artificial neural networks (ANN), which are one of the AI methods, have been used to identify and model illumination systems in several papers. Their use was considered in residential buildings in relation to residents' behavior patterns [4] or in classrooms [5]. In other studies, an office room lighting system with independently controlled dimmable LED luminaires was considered. Some of these studies assume no interaction with natural light. For instance, publications $[2,6]$ presents two-layer feedforward ANN lighting system model with 14 inputs and 12 outputs, used for optimization to achieve energy savings while providing personalized lighting control on occupied desks. Similarly, [7] shows two ANN models (two- and multilayer feedforward) and compares their performance with optimization based on a linear model. In turn, in [3],

*Corresponding author: mdechnik@pk.edu.pl 
an ANN model of a lighting system composed of 6 lamps and 6 desks was considered. Based on the network model, a closed-loop control system has been used, with a controller that uses the feedback from sensors to minimize energy consumption, including daylight.

This study relates to a lighting system with 28 individually dimmable lamps and 17 task tables in a large office room. Without knowledge about the relationships between the lamps' levels of dimming and the illuminances created on task desks, from a control point of view, the lighting system is a "black box". Using ANN, it is possible to identify these complex, both non-linear and linear dependencies by using function approximation [2].

\section{The need for personal (individual) illumination}

In indoor work environments, proper lighting favors the motivation of employees and increases their satisfaction with the work they do [8]. Human preferences of illuminance are quite different and vary depending on the type of work or their psycho-physical conditions [8]. For instance [9] shows that people chose illuminance between 110-1230 lx, while they had the possibility of lighting settings in private offices. Therefore, employees appreciate the possibility of individual adjustment of lighting conditions on their desks (personal control) [10]. In large rooms, with many workstations, one cannot have one illuminance that is suitable for all and permits optimization of energy use [11-13]. Local lighting control on the level of the desk introduces new concept of quality, enabling the adjustment of lighting conditions to individual preferences of people, as well as optimizing the energy efficiency of the lighting system [14].

\section{Lighting system identification using neural network}

In an open office space, light comes to one desk from different lamps and reflections of their light rays [2]. Therefore dimming changes made to one lamp do not control the illuminance at one desk, but it also affects the lighting of other neighboring desks. The area of a single lamp's impact is particularly large in European open-plan offices, which are characterized by a lack of partitions between desks (in contrast to the cubicle layout used in the US) [14]. In the European layout, traditional methods of controlling single luminaires or their groups do not work. For this reason, one must consider the whole lighting system and relate the system control to the illumination of individual desks. To this end we will examine application of the ANN.

In recent years, ANNs, inspired by the human nervous system, has been successfully applied in most branches of industry and science, and their development continues. These networks have the ability to perform a kind of "black box" analysis - a problem can be described on the basis of input and output data, without detailed knowledge of the internal dependencies of the system. In the first stage, the user prepares a representative set of examples of inputs and their corresponding outputs of the analyzed function, which constitute a pattern of proper network behavior. Then, during the ANN's learning process, the necessary data structure is automatically generated in the network's memory, and on its basis the network performs all functions related to the operation of the created model. ANNs are a modeling technique capable of approximating extremely complex functions.

The indoor space, about $100 \mathrm{~m}^{2}$, located at Malopolska Laboratory of Energy Efficient Building (Cracow University of Technology) was selected for this study. The room has the characteristics of modern offices - large floor to ceiling windows with exterior blinds, as well as a glazed partition wall. The interior was arranged as an open-plan office. Lighting is provided by a direct system consisting of 28 individually dimmable LED luminaires, and 17 task tables (Fig. 1). 


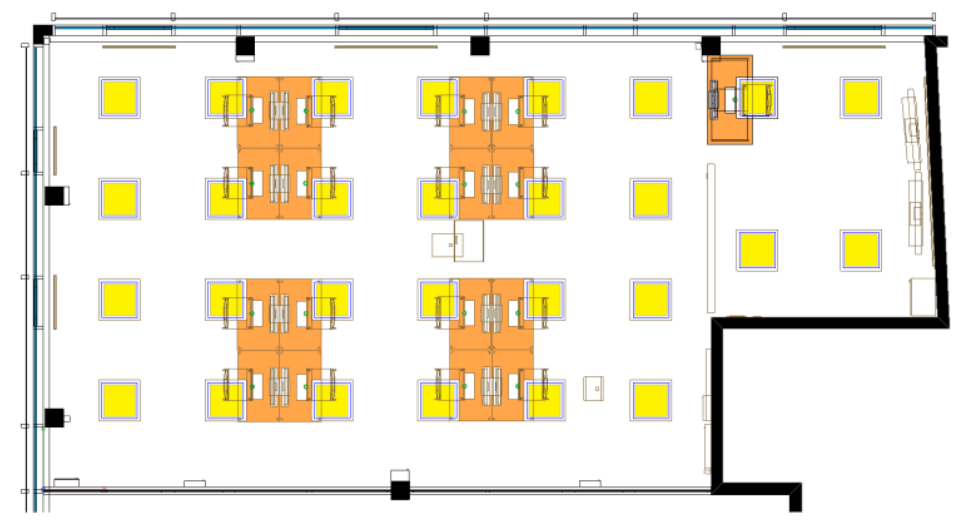

Fig. 1. LED luminaires (yellow) and task tables (orange) and chairs.

\subsection{Configuration of neural network}

The calculations were performed using Matlab R2011B version [15]. The input data for the ANN analysis were in this case 120 sets containing 28 inputs and 17 outputs. The inputs represented dimming levels of 28 luminaires in the range of 0 (lamp off) and from 0.1 to 1 (maximum luminous flux), while outputs illuminances on 17 task tables in the room (Fig. 1). For each of the sets the settings of the lamps were generated randomly and the resulting illuminances on the desks were calculated using DIALux 4.13 software based on the detailed computer model of the analysed room. In a future application of the model the information about illumination at the tables would be measured but in this case, we were interested if the ANN will agree with the used DIALux model.

Data processing was performed using a two-layer feedforward neural network implemented in Matlab. The feedforward type of neural network was chosen due to the scientific experience in the studied area described in $[2,6,7]$. The studies did not consider single-layer neural networks due to their limited capabilities. Usually, such structures are used for issues that do not require high computational complexity, for example in solutions to linearly separable problems [16]. For the study a functional fitting tool was used. The tool is described in detail in [16]. Figure 2 depicts the created neural network structure. This structure has one hidden layer consisting of 33 neurons and one output layer with 17 neurons. The number of neurons in output layer corresponds to the number of outputs. The number of neurons in hidden layer was experimentally selected. The condition for selecting the number of neurons in this layer was the value of correlation coefficient $(R)$ calculated for all data assigned for teaching greater than 0.99 . For the study there were no delays implemented on the input for any layer. The activation function for the hidden layer was tangensoidal (tansig). The choice of non linear activation function comes from the assumption that in the studied case, the occurrence of non-linearity is suspected [6]. The output layer had a linear activation function.

For the purpose of the ANN learning procedure, all input and output data were randomly assigned to 3 sets for the training, validation and testing stages, containing 70, 15 and $15 \%$ of data respectively. The Levenberg-Marquardt algorithm was chosen as the teaching algorithm, with training settings [15]: maximum number of epochs to train: 1000; performance goal: 0 ; learning rate: 0,01 ; maximum validation failures: 6 ; momentum: 0,9 ; minimum performance gradient: $10^{-10}$; maximum time to train in seconds: infinite. 


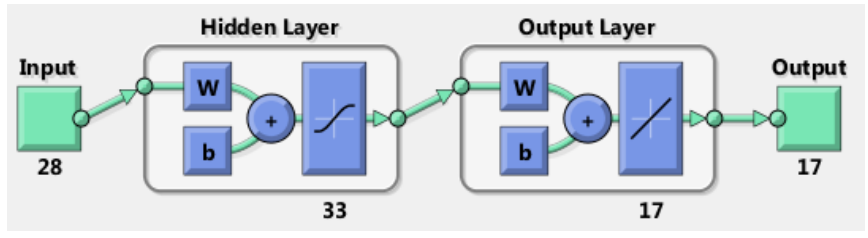

Fig. 2. The created ANN structure.

\subsection{Computation}

Figure 3 (left) depicts results obtained from the training, validation and test of the ANN in the form of an error histogram [16]. Figure 3 (right) presents the ANN performance graph during its learning. The ordinate axis refers to the ANN performance function values. Mean square error (MSE) was chosen as the performance function [16]. The horizontal axis corresponds to learning epochs. The system reached the best neural network validation of the ANN performance for the 73th epoch, and the minimum of performance function was equal to 1185,2593 . One can observe that the neural network system continued the learning algorithm for another 6 epochs in order to confirm the alleged local minimum for the goal set for the created network structure (Figure 2). From epoch 1 to 12, a downward trend in all groups of results of the ANN teaching procedure can be seen. It can also be seen that the downward trend for training data continued to the last epoch.
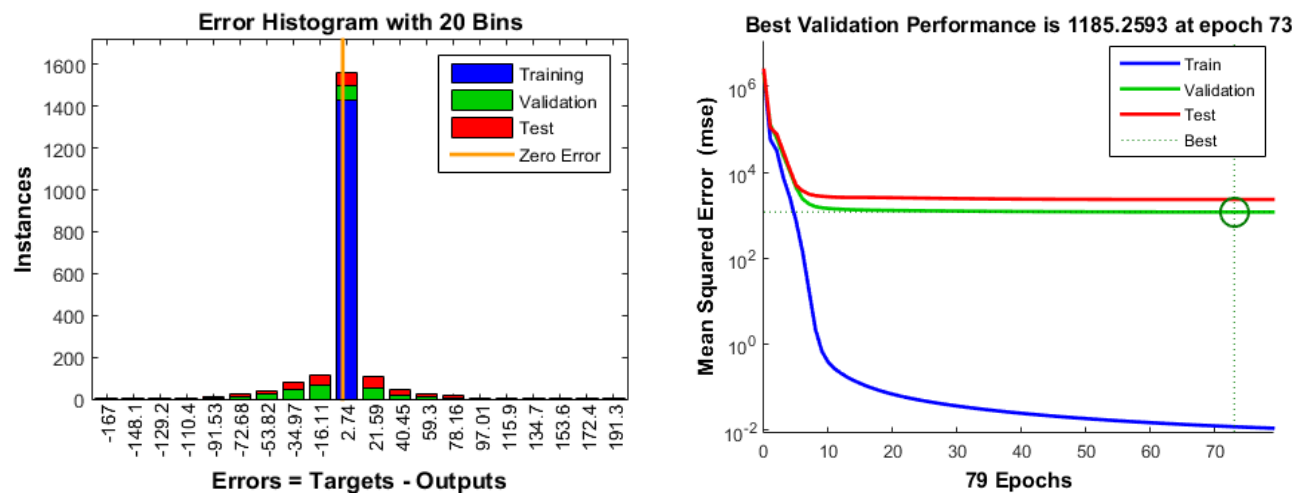

Fig. 3. Error histogram of the ANN (left) and performance of the ANN for successive learning epochs (right).

Figure 4 depicts the regression results for the training, validation and test and the regression for all data assigned to the ANN learning with a supervisor [16]. Here, the ordinate axis represents the neural network output for the given input data. The abscissa axis shows targets values. The $R=1$ regression result for all data means that there is an unequivocal relation between the actual value (targets from simulation) and the neural network output value. The regression results for the data assigned to the training, validation and testing stage were $R=1, R=0.98798$ and $R=0.97465$, respectively. Since $R$ has reached 1 for training stage, this indicates that the data assigned to the training stage should no longer be able to improve the weights of individual neurons in the network. All data regression coefficient $R$ equals to 0,99235 . This means that the results obtained at the ANN's output are very well matched to the data assigned to the learning process - the illuminances received from the neural network are almost consistent with the reference 
illuminances for corresponding dimming levels of lamps. As a result, ANN was trained to represent the analyzed actual lighting system.
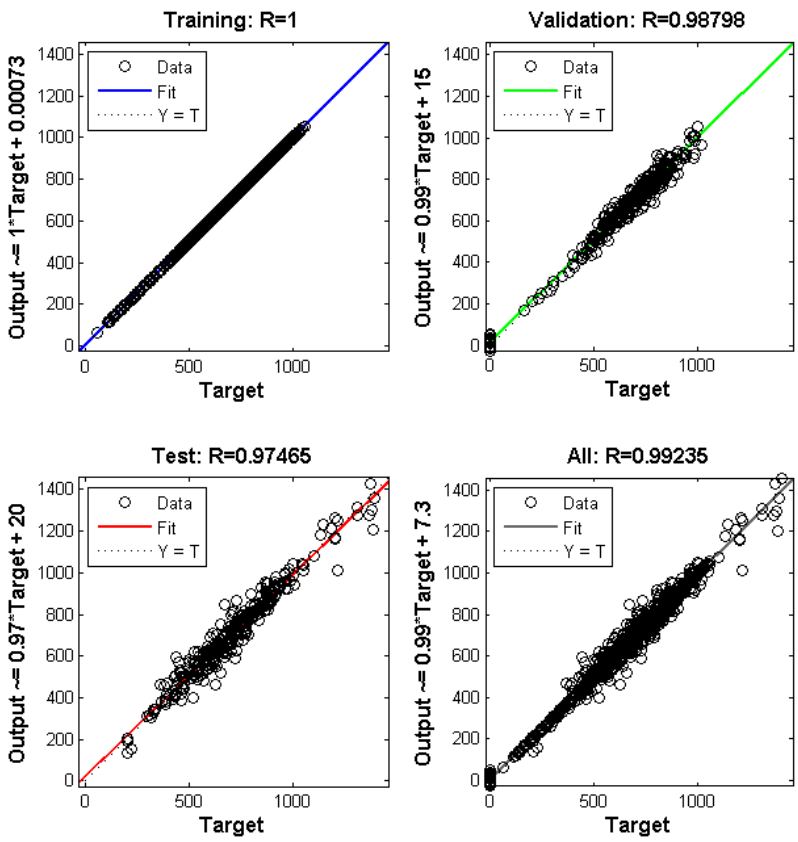

Fig. 4. Regression results for the training, validation and test and the regression for all data assigned to the ANN learning with a teacher.

\section{Concluding remarks}

The room lighting system was considered with 28 individually dimmable LED luminaires illuminating 17 task tables. A neural network mapping model has been developed, which shows promising results of approximation of dependencies occurring in the considered actual lighting system. This model can replace the actual room as a controlled object - to operate the lighting control system and create the desired lighting conditions, no light intensity sensors are needed on individual task desks. It is enough to perform measurements only once in order to collect data for network training. However, the disadvantage of ANN method is invalidation of the model, in the case of any change in the room affecting the light distribution - the input data collection and network training must be repeated. Furthermore, the challenge for the ANN method is the growing amount of data for network training along with the increased complexity of the system.

Use of ANN permits evaluation of a specific indoor environment and establishing relation between controlling the individual lamps and illumination conditions on a work table. Such systems have the potential to be used in open-plan offices due to the possibility of illumination rendering on individual task desks. The authors will continue this work to optimize individual lighting approach.

\section{References}

1. B. L. Ahn, C. Y. Jang, S. B. Leigh, S. Yoo, H. Jeong, Effect of LED lighting on the cooling and heating loads in office buildings, Appl. Energ., 113, 1484-1489 (2014) 
2. Z. Wang, Y. K. Tan, Illumination control of LED systems based on neural network model and energy optimization algorithm, Energ. Buildings, 62, 514-521 (2013)

3. N. K. Kandasamy, G. Karunagaran, C. Spanos, K. J. Tseng, B. H. Soong, Smart lighting system using ANN-IMC for personalized lighting control and daylight harvesting, Build. Environ., 139, 170-180 (2018)

4. A. Paulauskaite-Taraseviciene, N. Morkevicius, A. Janaviciute, A. Liutkevicius, A. Vrubliauskas, E. Kazanavicius, The Usage of Artificial Neural Networks for Intelligent Lighting Control Based on Resident's Behavioural Pattern, Elektron. Elektrotech., 21(2), 72-79 (2015)

5. Y. Chen, Q. Sun, Artificial intelligent control for indoor lighting basing on person number in classroom, 9th Asian Control Conference (ASCC), Istanbul (2013)

6. D. Tran, Y. K. Tan, Sensorless Illumination Control of a Networked LED-Lighting System Using Feedforward Neural Network, IEEE T. Ind. Electron., 61(4), 2113-2121 (2014)

7. V. Lobato-Ríos, V. del Rocío Hernández-Castañón, J. A. Carrasco-Ochoa, J. F. Martínez-Trinidad, Linear model optimizer vs Neural Networks: A comparison for improving the quality and saving of LED-Lighting control systems, 23rd International Conference on Pattern Recognition (ICPR), 2664-2669 (2016)

8. J. van Duijnhoven, C. de Bakker, M. P. J. Aarts, A. L. P. Rosemann, H. S. M. Kort, An unobtrusive practical method to derive individual's lighting conditions in office environments, 14th International Conference on Networking, Sensing and Control, IEEE, 471-475 (2017)

9. P. R. Boyce, N. H. Eklund, N. S. Simpson, Individual Lighting Control: Task Performance, Mood, and Illuminance, J. Illum. Eng. Soc., 29(1), 131-142 (2000)

10. J. A. Veitch; G. R. Newsham; P. R.Boyce; C. C. Jones, Lighting appraisal, well-being, and performance in open-plan offices: A linked mechanisms approach, Lighting Res. Technol., 40(2), 133-151 (2008)

11. A. Romańska-Zapała, M. Bomberg, M. Fedorczak-Cisak, M. Furtak, D. Yarbrough, M. Dechnik, Buildings with environmental quality management, part 2: Integration of hydronic heating/cooling with thermal mass, J. Build. Phys., 41(5), 397-417 (2018)

12. D. W. Yarbrough, M. Bomberg, A. Romańska-Zapała, Buildings with environmental quality management, part 3: From log houses to environmental quality management zero-energy buildings, J. Build. Phys. (online)

13. A. Romańska-Zapała, M. Bomberg, D. W. Yarbrough, Buildings with environmental quality management: Part 4: A path to the future NZEB, J. Build. Phys. (online)

14. C. de Bakker, M. B. C. Aries, H. S. M. Kort, A. L. P. Rosemann, Practical relevance of lighting control strategies in open-plan offices, INSide Information magazine TU/e, 06/2016, 16-17 (2016)

15. J. Bartman, Podstawowe funkcje biblioteki narzędziowej "Neural Network Toolbox. Version 5" pakietu MATLAB v.6, Uniwersytet Rzeszowski, Zakład Elektrotechniki i Informatyki, online

16. M. Dudzik, Contemporary methods of designing, validation and modeling of the phenomena of electrical traction; monograph, Cracow University of Technology, Kraków, Wydaw. PK (2018) 